\title{
PETRÓLEO Y DESARROLLO REGIONAL: EL CASO DE TABASCO*
}

\author{
María Eugenia Negrete Salas
}

\section{INTRODUCCIÓN}

LA REG IÓN ÍSTMICA DE MÉX ICO cuenta con recursos excepcionales para su desarrollo futuro. En particular, las planicies costeras del Golfo de México tienen abundante petróleo y otros recursos minerales, un potencial agrícola considerable, agua, energía eléctrica y en menor escala recursos forestales y pesqueros. Su localización geográfica coloca al istmo en situación de privilegio con respecto a los mayores mercados internos - Ciudad de México, Puebla y Guadalajara - y en óptimas condiciones de accesibilidad a los mercados externos del Atlántico y del Pacífico.

La presencia de un potencial como el descrito contrasta, sin embargo, con el bajo nivel de desarrollo en que se encuentra actualmente la zona. Los obstáculos más inminentes para el desarrollo de la región, en relación al sector agrícola, han sido la dificultad por controlar el agua de los ríos y drenar las tierras inundables, y la falta de tecnología adecuada para la explotación del trópico húmedo.

* Una primera versión de este trabajo fue presentada en el Convegno internazionale su: Intervento pubblico e sviluppo regionale. Trent'anni di esperienze. SASSARI, 27-31 Octubre, 1982. 
A la vez, el desarrollo industrial se ha visto limitado por la escasez de vías de comunicación que, hasta hace unos veinticinco años, caracterizó a la región, ${ }^{1}$ la falta de inversiones privadas y la poca calificación de la mano de obra en el lugar.

El istmo constituye una zona prioritaria para el desarrollo nacional. Las inversiones relacionadas con el petróleo se han multiplicado en años recientes: en Coatzacoalcos-Minatitlän se han incrementado las actividades industriales relacionadas con la petroquímica; en Salina Cruz y Coatzacoalcos se construyen puertos industriales y entre ambos puntos se proyecta la construcción de un puente terrestre que una las dos terminales (véase el mapa 1$)^{2}$

Pero hasta ahora, el impacto multiplicador de la inversión a nivel regional es mínimo, debido a los pocos vínculos intrarregionales y a que, gran parte de los bienes y servicios consumidos en la zona son importados.

El estado de Tabasco forma parte de la región ístmica; se compone de diecisiete municipios y tiene como capital la ciudad de Villahermosa (véase el mapa 2). Es un estado poco desarrollado con respecto al resto del país, algunos indicadores socioeconómicos para 1970 muestran que la entidad ocupaba el vigésimo lugar en cuanto a índice de desarrollo, ${ }^{3}$ el vigesimotercero en cuanto a valor agregado per cápita y que sólo $33 \%$ de su población vivía en localidades de más de 2500 habitantes.

Sin embargo, en la última década, las perspectivas de Tabasco han mejorado. La crisis interna de la economía mexicana, que obliga a replantear el modelo de desarrollo nacional, coincide con la crisis energética mundial y el descubrimiento de reservas cuantiosas de petróleo y gas natural en el subsuelo mexicano. Los hidrocarburos se convirtieron en instrumentos clave para el desarrollo futuro del país $y$, el hecho de que en territorio tabasqueño se localicen impor-

1 El ferrocarril del sureste, la carretera del Golfo y la carretera transístmica fueron construidos en la década de los cincuenta.

2 Para tener un panorama del desarrollo del Istmo véase International Bank for Reconstruction and Development, The economic development of the isthmic region of Mexico, Reporte núm. 1080-ME. Marzo, 1976, y Secretaría de Asentamientos Humanos y Obras Públicas, Plan nacional de desarrollo urbano. Plan regional de desarrollo urbano zona prioritaria costera del Golfo e Istmo de Tehuantepec, versión abreviada, mayo, 1976.

3 Calculado de acuerdo con la información censal y jerarquizando las 32 entidades de la República Mexicana. International Bank for Reconstruction and Development, op. cit., p. 1. 
Mapa 1

Localización del estado de Tabasco, México

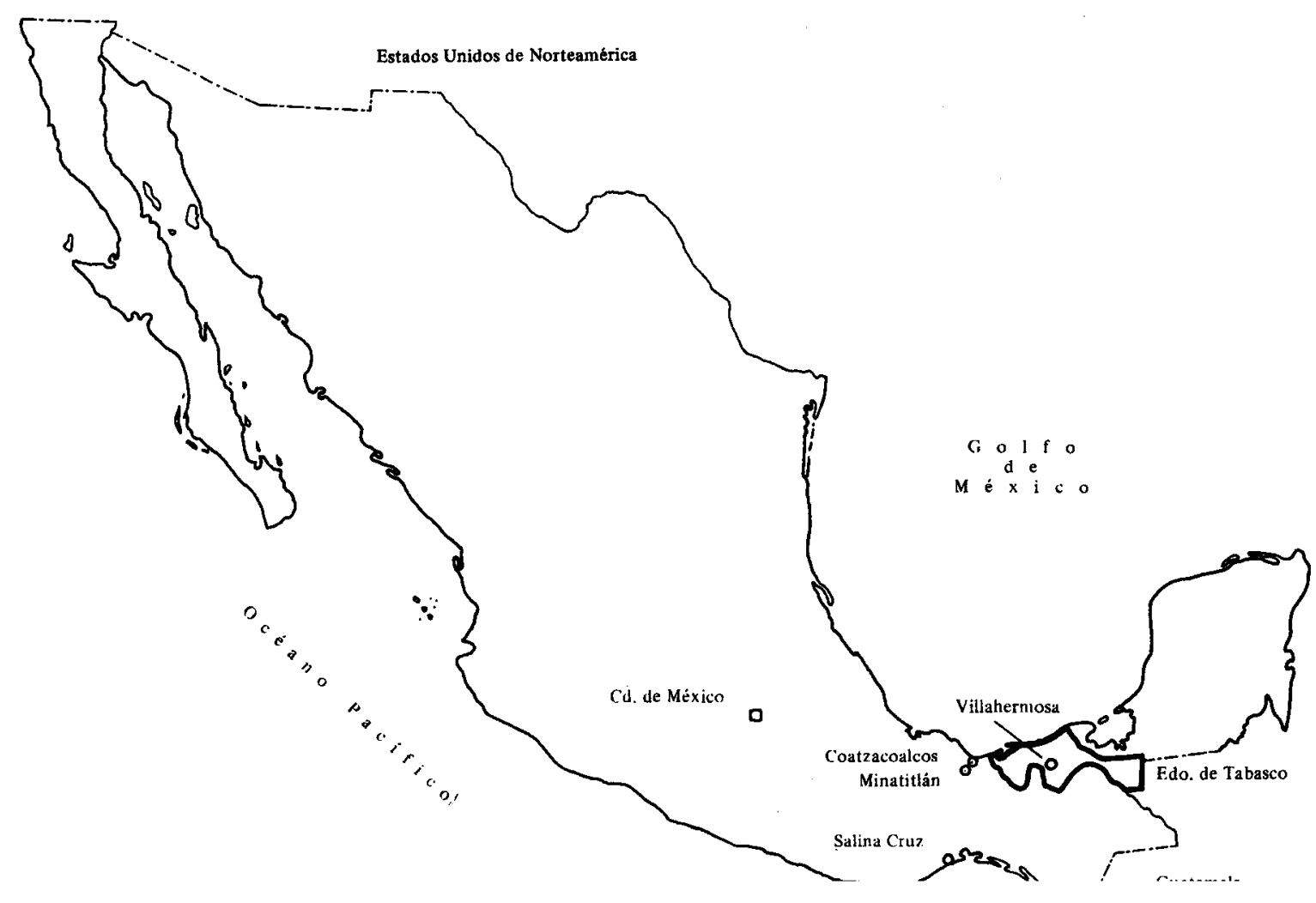


Municipios del estado de 1 abasco

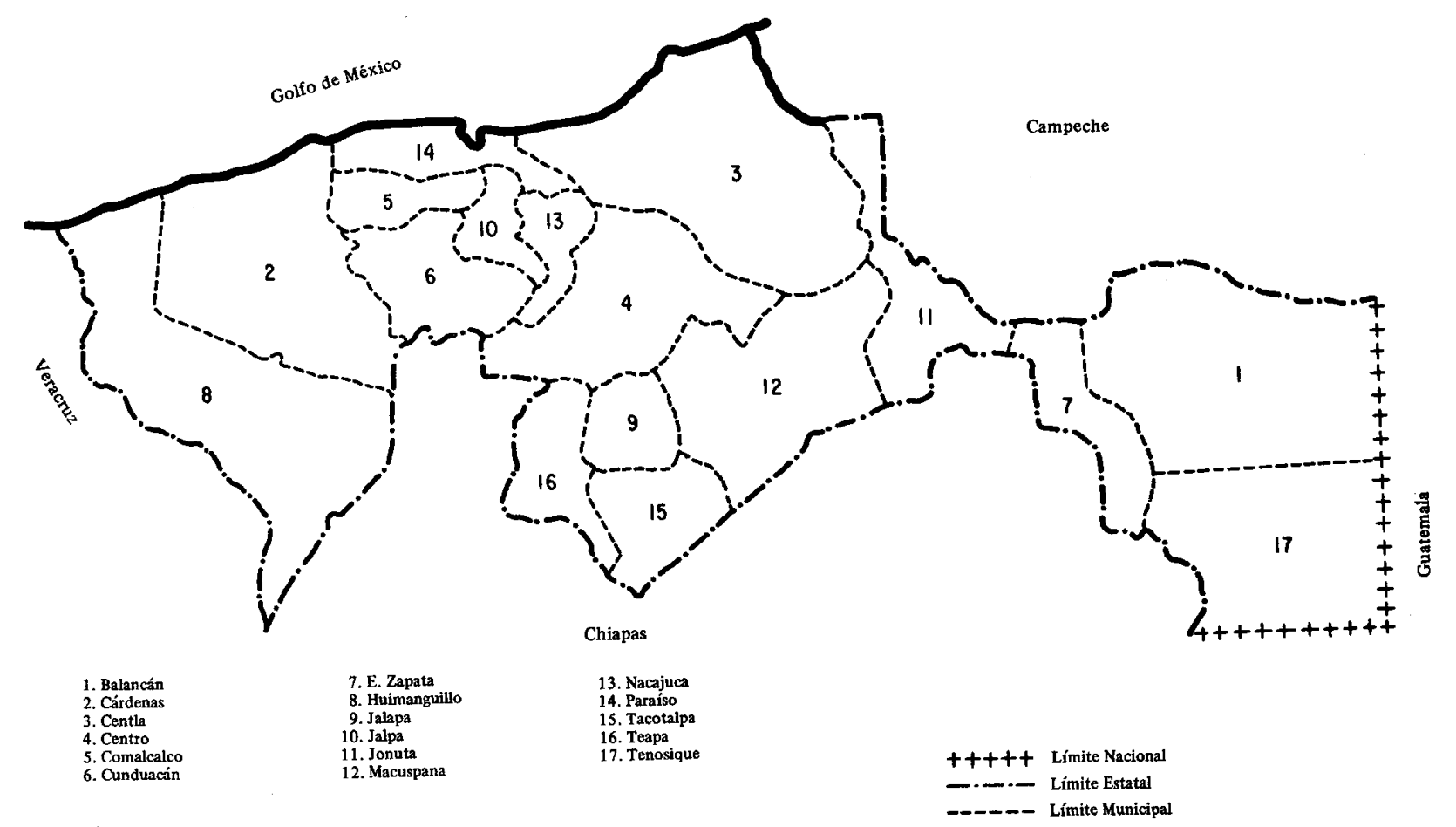


tantes yacimientos, vuelve al estado un foco de atención importante dentro del marco de la planeación económica nacional.

En la primera parte de este documento se presenta un análisis de la evolución económica y demográfica reciente en Tabasco, luego se analiza el papel del petróleo como impulsor de la economía nacional y por último se discuten las perspectivas del desarrollo regional de Tabasco, en el contexto de la planificación económico-espacial del país.

\section{EVOLUCIÓN ECONÓMICO-DEMOGRÁFICA EN TABASCO (1940 a 1980)}

A principios de este siglo, la estructura productiva en el estado se caracterizaba por una actividad de tipo plantación para la producción y exportación de plátano, a través de compañias norteamericanas. A partir de la década 1930 a 1940 entra en crisis esta producción y se inicia en el estado una actividad agrícola y ganadera cada vez más importante, así como el desarrollo de una planta agroindustrial.

Este impulso económico se basó en acciones orientadas al mejoramiento de las condiciones productivas del sector primario y a un desarrollo paulatino de la actividad secundaria: La acción más importante fue, sin duda, la emprendida con el "Plan Chontalpa" iniciado en 1965. Este plan buscaba la incorporáción a la producción de 91000 hectáreas en el estado de Tabasco que quedaron libres de inundaciones con la construcción de la presa Nezahualcóyotl. La ejecución del plan ha sido costosa y sus distintos programas han tropezado con enormes dificultades. ${ }^{4}$ A pesar de que los resultados obtenidos son poco satisfactorios, el plan sigue apoyado por el gobierno, pues constituye una experiencia piloto del uso de tecnología adecuada para la explotación del trópico húmedo y de las potencialidades de la colectivización del ejido.

El descubrimiento reciente (1973 a 1976) de extensos yacimientos petroleros y su explotación, indujo a un cambio económico importante que modificó las tendencia del desarrollo socioeconómico en el estado y cuyas características se describirán más adelante.

Entre 1940 y 1980 casi se cuadruplicó la población de Tabasco, pasando de 285630 a 1062961 habitantes. Este crecimiento se dio a un ritmo -similar al que se dio en todo el país- hasta 1960. Pero

4 Barkin, David, Desarrollo regional y reorganización campesina: La Chontalpa como reflejo del problema agropecuario mexicano, Centro de Ecodesarrollo, Nueva Imagen, México, 1978. 
a partir de ahí y hasta 1980, la población del estado aumentó a tasas de $4.6 \%$ y $4 \%$, mientras el resto del país lo hizo a $3.4 \%$ y $3.3 \%$ sucesivamente (véase el cuadro 1 ).

El componente natural del crecimiento de Tabasco ha sido superior al nacional; esto es el resultado de tasas de mortalidad mucho menores y de tasas de natalidad ligeramente inferiores al promedio de México. Durante este período, se registraron fuertes descensos en los niveles de mortalidad (principalmente en la mortalidad infantil) de 14.5 muertes por cada 1000 habitantes a sólo 6.3, en 1976. Por otra parte, la natalidad aumentó de 38 nacimientos por cada 1000 habitantes en 1940 hasta 46.7 en 1965 y; a partir de ese año, desciende lentamente hasta alcanzar los 40.2 nacimientos por 1000 habitantes en 1975. El resultado de estos cambios llevó al estado de

\section{Cuadro 1}

República mexicana y Tabasco: Población total y tasa de crecimiento medio anual, 1940 a 1980

\begin{tabular}{|c|c|c|c|c|}
\hline$A \bar{n} o$ & $\begin{array}{l}\text { Repúblico } \\
\text { Población }\end{array}$ & $\begin{array}{l}\text { exicana } \\
\text { Tasa de } \\
\text { crecimiento } \\
\text { anual }\end{array}$ & Población & $\begin{array}{c}\text { Tasa de } \\
\text { crecimiento } \\
\text { anual }\end{array}$ \\
\hline 1940 & 19653552 & 2.7 & 285630 & 2.4 \\
\hline 1950 & 25791017 & 3.1 & 362716 & 3.2 \\
\hline \multirow[t]{2}{*}{1960} & 34923129 & & 496340 & \\
\hline & & 3.4 & & 4.6 \\
\hline \multirow[t]{2}{*}{1970} & 50694617 & & 811114 & \\
\hline & & 3.3 & & 4.0 \\
\hline 1980 & 69346900 & & 1062961 & \\
\hline
\end{tabular}

Fuente: Consejo Nacional de Población. México Demográfico, Breviario 1980-1981 y X Censo de Población y Vivienda, 1980, estado de Tabasco. 
Tabasco a registrar una tasa de crecimiento natural, ascendente hasta 1975 y descendente a partir de ese punto de inflexión (véase el cuadro 2).

\section{Cuadro 2}

Tabasco: indicadores demográficos, 1940 a $1975^{*}$

\begin{tabular}{lcccccc}
\hline $\begin{array}{l}\text { Indicadores } \\
\text { demográficos }\end{array}$ & 1940 & 1950 & 1960 & 1965 & 1970 & 1975 \\
\hline $\begin{array}{l}\text { Tasa bruta de } \\
\text { natalidad }\end{array}$ & 38.4 & 43.8 & 45.2 & 46.7 & 42.7 & 40.2 \\
$\begin{array}{l}\text { Tasa bruta de } \\
\text { mortalidad }\end{array}$ & 14.5 & 12.6 & 9.5 & 8.0 & 8.3 & 6.5 \\
$\begin{array}{l}\text { Tasa de crecimiento } \\
\text { natural }\end{array}$ & 23.9 & 31.2 & 35.7 & 38.7 & 34.4 & 33.9 \\
\hline
\end{tabular}

- Tasa por mil.

Fuente: Consejo Nacional de Población, Diagnóstico y politica demográfica del estado de Tabasco, 1981 (mimeog.).

El crecimiento debido a las migraciones se ha comportado de manera peculiar. A partir de los años 60 , coincidiendo con el mejoramiento en las comunicaciones y la consecuente integración al mercado nacional, empieza a manifestarse con relativa importancia el crecimiento social, y Tabasco se convierte en una entidad federativa de "débil atracción". 5 Los estados que envía migrantes a Tabasco son principalmente: Campeche, Chiapas, Distrito Federal, Oaxaca, Veracruz y Yucatán.

La migración intraestatal ha jugado un papel importante en el proceso de urbanización pues la población urbana creció a tasas su-

5 En función de sus tasas de crecimiento social, las entidades federativas han sido clasificadas por el Consejo Nacional de Población en: entidades de fuerte atracción (tasas superiores a $1 \%$ anual); entidades de debil atracción (tasas entre $0.6 \%$ y $1 \%$ anual); entidades de equilibrio (tasas alrededor de $0 \%$ ); entidades de débil expulsión (tasas negativas entre $-0.6 \%$ y $-1 \%$ anual) y entidades de fuerte expulsión (tasas negativas inferiores $a-1 \%$ ). 
periores a las de la población rural, especialmente a partir de 1960 . Entre ese año y 1970, se duplicó la población de Villahermosa que pasó de 55360 a 103694 habitantes, mientras que Ciudad Cárdenas casi la cuadruplicó al aumentar de 4583 a 16117 habitantes.

La orientación que se observa en los flujos migratorios en el estado entre 1940 y 1970 está estrechamente relacionada con el empleo en los distintos sectores económicos, pues los migrantes se dirigen hacia los municipios donde predominan las actividades con mayor capacidad de absorción de fuerza de trabajo. Hasta 1970, tanto los migrantes provenientes de otros estados de la República como los originarios de Tabasco, se dirigian principalmente hacia los municipios donde se desarrollaban actividades agropecuarias (véase el cuadro 3 ). Los municipios que expulsaron población presentan decrementos en la población ocupada en actividades primarias y secundarias y sólo el terciario incrementó ligeramente su capacidad de absorción de fuerza de trabajo.

En la década 1970 a 1980 se modifican las características de la migración. Los cambios se manifiestan tanto en la intensidad como en la dirección de los flujos migratorios, pues la población se desplaza hacia los municipios donde se concentra la actividad relacionada con PEMEX.

Los datos agregados con respecto a la evolución económica del estado muestran cómo, en el último medio siglo, la actividad del sector primario ha decaido paulatinamente $y$ en la actualidad ha perdido su carácter predominante. Después de haber ocupado $80.66 \%$ de la población económicamente activa en 1940, pasó a ocupar sólo $42.7 \%$ en 1980 (véase el cuadro 4). Esta disminución se ha compensado con el incremento en las actividades urbanas (secundaria y terciaria) en el estado, pues ambos sectores aumentaron la proporción de población ocupada en el mismo período. La ocupación en el sector secundario se triplicó mientras el terciario, con un ritmo ligeramente más lento, pasó de ocupar $13.23 \%$ de la población económicamente activa en 1940 a $32.8 \%$ en 1980 .

Dentro de la producción del sector primario, la ganadería ha incrementado su participación en detrimento de la agricultura, lo cual, seguramente ha influido en la disminución de la capacidad de absorción de fuerza de trabajo en este sector.

En términos del producto interno bruto estatal (PIB), la participación de los sectores muestra aún más acentuada esta tendencia (véase el cuadro 5). La participación relativa del sector primario descendió, al pasar de $76.9 \%$ en 1940 a sólo $13.84 \%$ en 1970 . En este lapso la actividad petrolera jugó un papel importante impul- 
Cuadro 3

Tabasco: Municipios de atracción, equilibrio y rechazo, 1960 a 1980 .

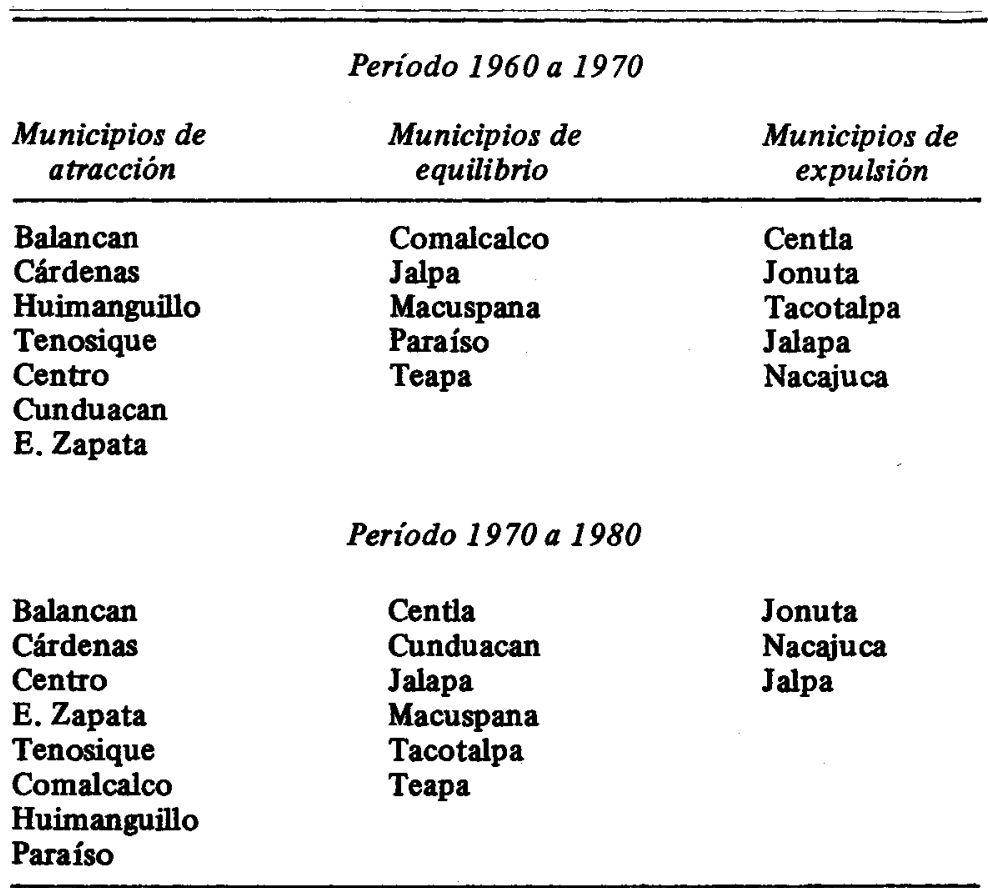

Fuente: Consejo Nacional de Población. Diagnóstico y politica demográfica del estado de Tabasco, 1980.

sando las actividades secundarias, ya que este sector aumentó su participación de $4.70 \%$ a $47.45 \%$. Por su parte, el terciario manifestó una tendencia a incrementar su participación en el PIB estatal, pasando de $18.31 \%$ a $38.71 \%$.

Los datos anteriores son un indicador elocuente del descenso relativo de la productividad agrícola ${ }^{6}$ y del incremento simultáneo

6 A pesar de que la producción del sector agropecuario descendió en términos relativos, la población ocupada se duplicô pasando de 62105 en 1940 a 122049 en 1970. En los otros dos sectores sucedió lo contrario. 
Negrete: Petróleo y deSARrollo Regional

\section{Cuadro 4}

Tabasco: PEA de 12 años y más por sector económico, 1940 a 1980 (porcentajes)

\begin{tabular}{lrrrrr}
\hline & 1940 & 1950 & 1960 & 1970 & 1980 \\
& $100 \%$ & $100 \%$ & $100 \%$ & $100 \%$ & $100 \%$ \\
\hline Sectal & 80.66 & 74.5 & 70.9 & 62.0 & 42.7 \\
$\begin{array}{l}\text { Secundario } \\
\text { (incluye petróleo) }\end{array}$ & 6.11 & 9.6 & 11.2 & 14.2 & 20.7 \\
$\begin{array}{l}\text { Terciario } \\
\text { Otros }\end{array}$ & 13.23 & 15.9 & 17.9 & 23.8 & 32.8 \\
\hline
\end{tabular}

Fuente: Censos de Población y Consejo Nacional de Población, Encuesta sociodemográfica de Tabasco, CONAPO-COPRODET, 1980.

en la del trabajo en las actividades secundarias. La información relativa al comercio y a los servicios, revela por el contrario un ascenso de la productividad en este sector. Este esbozo de la evolución económica, que se ha llevado a cabo en Tabasco, es útil para explicar la lógica de las migraciones y de la distribución de la población en el territorio tabasqueño.

\section{Petróleo y desarrollo en México}

La existencia de importantes reservas de petróleo y gas natural en territorio nacional ${ }^{7}$ representa la posibilidad real de modificar el modelo de desarrollo del país, basado en la sustitución de importaciones. Dicho modelo fue relativamente exitoso al permitir, en el

7 Las reservas probadas llegaron en 1980 a 50022 millones de barriles que, al ritmo de producción de ese momento, tenían una duración aproximada de 64 años. Secretaría de Programación y Presupuesto, Las actividades económicas en México, 1980, Seria manuales de información básica de la nación, tomo 3, 1980. 
Cuadro 5

Tabasco: PIB por sectores, 1940 a 1970 (millones de pesos 1950)

\begin{tabular}{|c|c|c|c|c|c|c|c|}
\hline \multirow[b]{2}{*}{$A \bar{n} o$} & \multirow{2}{*}{$\begin{array}{c}P I B \\
\text { total }\end{array}$} & \multicolumn{2}{|c|}{$\begin{array}{l}\text { Sector } \\
\text { primario }\end{array}$} & \multicolumn{2}{|c|}{$\begin{array}{l}\text { Sector } \\
\text { secundario }\end{array}$} & \multicolumn{2}{|c|}{$\begin{array}{l}\text { Sector } \\
\text { terciario }\end{array}$} \\
\hline & & Absolutos & $\%$ & Absolutos & $q$ & Absolutos & $\%$ \\
\hline 1940 & 21635 & 16654 & 76.97 & 1018 & 4.70 & 3963 & 18.31 \\
\hline 1950 & 33136 & 17793 & 53.70 & 2244 & 6.77 & 13099 & 39.53 \\
\hline 1960 & 76337 & 25865 & 33.88 & 30966 & 40.57 & 19506 & 25.55 \\
\hline 1970 & 170451 & 23593 & 13.84 & 80883 & 47.45 & 65975 & 38.71 \\
\hline
\end{tabular}

Fuente: Consejo Nacional de Población. Diagnóstico y política demográfica del estado de Tabasco, 1981 (mimeog.) 
período 1940 a 1970 , tasas de crecimiento del producto interno bruto del orden de $6 \%$ anual en promedio ${ }^{8}$ y al impulsar la instalación de una planta industrial, orientada a la producción de bienes de consumo.

El costo de crecimiento fue alto para los mexicanos y también para el país. La dinámica económica de México se acompañó de un fenómeno creciente de polarización de las desigualdades: desequilibrios intra e intersectoriales; distribución del ingreso y del consumo; desigualdades regionales; desempleo y subempleo, etc. Por otra parte, al subordinar la importancia de las exportaciones, la sustitución de importaciones generó una barrera estructural al crecimiento, basada en la escasez de divisas.

Durante los dos últimos sexenios, han aparecido los síntomas de la crisis, inherentes al agotamiento del modelo: creciente endeudamiento externo, inflación, deterioro de las finanzas públicas, devaluación del peso, etc. Ambos gobiernos, el de Echeverría y el de López Portillo, reconocieron abiertamente estos problemas y pusieron en práctica medidas que, si bien hasta el momento pueden calificarse de poco exitosas, intentaban sanear la economía apoyándose en las posibilidades de las exportaciones de hidrocarburos.

Mucho se ha discutido sobre si el modelo de desarrollo basado en el petróleo podrá o no superar los problemas típicos del subdesarrollo al permitir, en principio, eliminar la barrera de la escasez de divisas, posibilitar el saneamiento de las finanzas públicas, fomentar la industrialización y crecimiento acelerados y abrir opciones de redistribución. Los distintos puntos de vista, algunos optimistas y otros no tanto, ${ }^{9}$ coinciden en alertar sobre los peligros que implica

8 Este ritmo no fue uniforme, presentó notables fluctuaciones. Véase Clark Reynolds, The mexican economy, twentieth century; structure and growth. New Haven, Yale University Press, 1970.

9 Las visiones optimistas se basan en aspectos que consideran favorables para lograr, con el petróleo, un verdadero impulso al desarrollo económico: antecedentes de industrialización en el país; estabilidad política y poder negociador del petróleo en las relaciones internacionales, etc. Véase René Villarreal, El petróleo como indstrumento de desarrollo: México en los 80's, Cuadernos sobre prospectiva energética, núm. 1, México, El Colegio de México, 1980. La opinión contraria, más bien pesimista, se sustenta en los antecedentes de desarrollo en México donde los que la apoyan ven que el gobierno manifiesta su incapacidad para prever los problemas y lograr un desarrollo más equilibrado. Véase Antonio Yúnez Naude, "Política petrolera y perspectivas de desarrollo de la economía mexicana, un ensayo exploratorio", Las perspectivas del petróleo mexicano, Centro de Estudios Internacionales, México, El Colegio de México, 1979. 
para México el desarrollo, basado en un recurso no renovable como el petróleo. El problema clave será convertir los dólares que ingresan por el petróleo en ahorro interno e inversión productiva, contrarrestando la tendencia al consumo y disminuyendo las presiones inflacionarias.

Otro aspecto esencial es la dependencia derivada de la monoexportación, por lo que se proponen medidas para hacer crecer la capacidad de respuesta interna frente a los vaivenes del mercado mundial, especialmente el de energéticos. Esto se haría básicamente mediante programas equilibrados de desarrollo sectorial y orientados a buscar una mayor competitividad internacional de las manufacturas mexicanas, por medio de la sustitución de importaciones de bienes de capital y de la autosuficiencia en productos agropecuarios y alimentos.

A juzgar por la experiencia reciente, las expectativas sobre el "auge petrolero" se han reducido y se acercan más a la duda y la frustración que a la esperanza. Pero cualquiera que sea el grado de desarrollo económico que se alcance en los próximos años, dos aspectos esenciales quedan claros: que este desarrollo estará basado, primordialmente en los recursos de hidrocarburos y que el Estado no podrá eludir una responsabilidad creciente en el manejo de la economía nacional.

Para efectos de esta discusión, cabe preguntarse ¿qué significan, en términos de desarrollo regional, las características del nuevo modelo mexicano basado en la exportación de hidrocarburos?, ¿cuáles son sus implicaciones espaciales?

El modelo sustitutivo de importaciones, al menos en sus etapas iniciales, organizó los mercados de trabajo concentrando la población y los recursos para la industrialización en pocas ciudades. El proceso se acompañó de migraciones masivas del campo a la ciudad y de diferencias, cada vez mayores, en las condiciones de empleo, vivienda, salud, educación, etc., en las ciudades y las zonas rurales.

Vista en términos de su organización espacial, la estructura de la economía mexicana hasta 1970 se vio determinada, en primer lugar, por la superconcentración en la ciudad de México y, en segundo, por la cercanía y el poder económico de Estados Unidos. Las características esenciales de esta estructuración espacial son: la constitución de un sistema de ciudades interdependientes pero fuertemente "dominado" por la concentración de la capital, la cual se erige en nodo central, comunicado con el resto del país y con la economia norteamericana y una diferenciación creciente entre las distintas regiones 
del país y entre cada ciudad y su periferia rural. En la dimensión político-administrativa, esta estructura implicó el establecimiento de relaciones tipo "centro-periferia" y por consiguiente, una distribución geográficamente desigual de los beneficios económicos.

Estas eran las consecuencias lógicas del modelo adoptado y, concidiendo con su virtual agotamiento, el gobierno mexicano a partir de 1970, manifestó, cada vez más enfáticamente, su deseo de descentralizar la economía, de desarrollar eficientemente los recursos de la periferia y de reducir la desigualdad entre los sectores rural y urbano.

En este contexto, la planificación regional buscó disminuir las diferencias, promoviendo el desarrollo de regiones y basándose en una descentralización de la industria. ${ }^{10}$ Se pensó que este proceso constituiría una segunda etapa en la sustitución de importaciones, originada en el incremento de la demanda del centro de materias primas de la periferia y en el desarrollo de fuerzas políticas locales, relacionadas con los mercados regionales." ${ }^{11}$ A pesar de las medidas de apoyo al desarrollo regional, esta inercia descentralizadora ha sido incipiente, y sólo cuando hubo grandes inversiones como en el caso del desarrollo de cuencas hidrológicas o del desarrollo siderúrgico de "Las Truchas", se logró un cierto avance. ${ }^{12}$

El desarrollo basado en el petróleo tiene, en principio, efectos directos de descentralización. Es obvio que la actividad petrolera implica una canalización de inversiones y recursos hacia la región de explotación, pero un segundo efecto se relaciona con el procesamiento del petróleo crudo y el acceso a los mercados internacionales.

10 Las medidas más importantes que se han tomado en este sentido fueron: la Ley de industrias nuevas y necesarias (1941), el Programa de parques industriales (1953), el Programa para la promoción de conjuntos, parques, ciudades industriales y centros comerciales SOP-NAFINSA (1971), los Decretos de descentralización industrial (1971), y el Programa de estímulos para la desconcentración territorial de las actividades industriales PRODETAP (1979). Véase Gustavo Garza. "Desarrollo económico, urbanización y políticas urbanoregionales en México (1900-1982), Demografía y Economia, El Colegio de México, vol. XVII, núm. 1 (54), 1983.

11 Véase Unikel, Luis, "Ensayo sobre políticas de desarrollo regional en México", Revista Interamericana de Planificación, SIAP, vol. 10, núm. 37, México, 1976.

12 Véase Barkin, D., Los beneficiarios del desarrollo regional, SepSetentas núm. 52, 1972; Barkin D. y King T. Desarrollo económico regional: (enfoque por cuencas hidrológicas de México), México, Siglo XXI Editores, 1975;Zapata F. et al., Las truchas. Acero y Sociedad en México, Centro de Estudios Sociológicos, El Colegio de México, 1978. 
En la medida en que se desarrollen los complejos petroquímicos; las grandes refinerías y depósitos, los oleoductos y gasoductos, así como la infraestructura portuaria que requieren las exportaciones, se modificarán los patrones espaciales tradicionales sobre el territorio nacional.

\section{EL desarRollo Regional EN México y LAS PERSPECTIVAS DEL ESTADO DE TABASCO}

En México las políticas de desarrollo regional se multiplican a partir de los años setenta. Si bien el aspecto espacial no estaba del todo ausente en las políticas económicas adoptadas, e incluso se habían puesto en práctica planes para el desarrollo de regiones especificas, es en el último decenio cuando se consolida un importante aparato planificador que explícitamente considera la dimensión territorial del desarrollo. ${ }^{13}$

Los principales objetivos de desarrollo regional, expresados en la mayor parte de los planes, pueden sintetizarse en tres puntos:

- Contribuir al mejor uso y explotación de los recursos naturales y por lo tanto al incremento de la producción.

- Buscar la descentralización demográfica y de actividades económicas para aliviar la concentración de las ciudades de México, Guadalajara y Monterrey y procurar la atracción de la población hacia otras regiones (costas y frontera).

- Mejorar las relaciones campo-ciudad para facilitar la expansión del desarrollo de las zonas urbanas.

Para alcanzar las metas propuestas, se considera a la industrialización como el medio más eficaz y se proponen estímulos de tipo fiscal para fomentar la "desconcentración concentrada" de la industria. Paralelamente, se apoya un programa de desconcentración del gasto público que eleve significativamente la inversión pública estatal.

La primera de las medidas ha probado reiteradamente su incapacidad para promover el desarrollo en las zonas de desconcentra-

13 Garza, Gustavo, op. cit. 
ción, ${ }^{14}$ mientras que la segunda se perfila como principal impulsor del desarrollo futuro de los estados.

Para llevar a cabo la descentralización del gasto público, se diseñaron instrumentos jurídico-administrativos, de los cuales mencionaré solamente los más importantes:

- Los comités promotores del desarrollo socioeconómico de los estados de la República (COPRODES), cuyo trabajo se basa en los programas de inversión pública federal a nivel de estados $y$,

- los convenios únicos de coordinación (CUC) entre el ejecutivo federal y los gobernadores de los estados.

- Asimismo, dentro del Plan Nacional de Desarrollo Urbano, se han diseñado diversos programas llamados de "acción concertada", para apoyar la desconcentración territorial de la administración pública federal; programa de estímulos para la desconcentración de las actividades industriales; programa de dotación de infraestructura de apoyo a puertos industriales; programa de dotación de infraestructura de apoyo a los energéticos, etc.

Es indudable la importancia de todas estas medidas, sin embargo, la evaluación con respecto a la eficacia de este aparato planificador desde el punto de vista de los objetivos originales, es aún prematura. En cuanto al futuro próximo, si tomamos en cuenta la crisis actual por la que atraviesa el país, puede pensarse que las metas de desarrollo regional, al menos en el corto plazo, se verán subordinadas más enfáticamente que antes a las metas de eficiencia nacional. ${ }^{15} \mathrm{~A}$ pesar de las condiciones adversas, la eventual superación de la crisis y el desarrollo nacional, se basarán en los recursos energéticos, por lo que de cualquier forma, la zona del sureste y en particular el estado de Tabasco seguirán siendo prioritarios.

14 Véase Unikel L. y A. Lavell, "El problema urbano-regional en México", Gaceta UNAM, Cuarta Época, vol. 3, núm. 20, suplemento, 9 de agosto, México, 1979.

15 Las condiciones sociales y políticas del país llevan a pensar que es difícil que los objetivos de redistribución social y territorial desaparezcan de la política económica a pesar de que el crecimiento económico se detenga por la crisis actual; será en todo caso cuestión de disminuir el énfasis en esas medidas. 


\section{Impacto de la actividad petrolera en Tabasco}

En lo que sigue de este documento se exponen muy brevemente los aspectos sobresalientes, producto de la actividad petrolera reciente en el estado de Tabasco, para tener algunos elementos de discusión acerca del futuro desarrollo de esta entidad.

A casi diez años de los descubrimientos de yacimientos de petróleo y gas natural en Tabasco, la actividad de PEMEX en el estado ha traído consigo los siguientes efectos:

1. En el aspecto demográfico, elevó la capacidad de atracción de población de la entidad, provocando inmigración desde otros estados y modificando, en lo interno, la intensidad y orientación de la migración. La población se dirigió hacia los municipios donde se concentra la actividad petrolera (explotación, almacenamiento y transporte) y hacia las localidades urbanas (véase el cuadro 1).

2. En el aspecto de ocupación, las nuevas actividades generaron pocos empleos directos. Hasta 1979, PEMEX demandaba 18400 empleos directos, de los cuales 7000 eran fijos y 11400 transitorios, pues la mayor parte de estos trabajadores se emplearon en trabajos de construcción. Los puestos administrativos y técnicos, que requieren una mayor calificación, fueron ocupados por migrantes, principalmente del Distrito Federal y Veracruz.

Esto se debió a la organización de Petróleos Mexicanos, cuyo centro administrativo y técnico se localiza en el Distrito Federal, y se reforzó con la poca calificación de la mano de obra local. ${ }^{16}$

3. Se han acentuado las diferencias salariales entre las distintas ramas de actividad, debido a que las remuneraciones pagadas por PEMEX son superiores a las que se perciben en otras actividades. E1 cuadro 6, muestra con claridad estas tendencias. Los ingresos inferiores al salario mínimo se concentraron marcadamente en la agricultura mientras que en la industria petrolera fueron prácticamente inexistentes. Asimismo, los ingresos en la construcción no relacionada con PEMEX y en las actividades de servicios fueron muy bajos.

En el extremo opuesto, se puede ver cómo los ingresos tres veces superiores al salario mínimo presentaron una concentración significativa sólo en la industria y en la construcción de PEMEX. La población

16 Váase Marie France Shapira, "Les travailleurs du pétrole", Cahiers des Ameriques Latine, núm. 20, 1979 y Secretaría de Programación y Presupuesto, Información sobre gasto público, 1969-1978. 
ocupada en la transformación se distribuyó equitativamente entre los grupos de ingreso, inferior a tres veces el salario mínimo.

Esta situación distorsionó la economía local, pues por una parte elevó los salarios en las otras ramas de actividad, desalentando las inversiones locales, y por otra incrementó la demanda de bienes y servicios, no producidos localmente, y encareció los que sí se producen en la zona.

4. Una consecuencia importante para Tabasco, es el impacto que la actividad petrolera ha tenido en los ingresos estatales. Antes de 1973, el ingreso de Tabasco por concepto de producción petrolera era de 1.5 millones de pesos, pero en 1974 subió a cerca de 14 millones; para 1975 se incrementó a 150 y en 1977 superó los 650 millones de pesos. ${ }^{17} \mathrm{La}$ intensidad y rapidez de este cambio ha requerido grandes esfuerzos por parte de la administración pública local. Los recursos se han canalizado fundamentalmente a la realización de obras públicas y de infraestructura, requeridas por las nuevas actividades y la atención a otros sectores ha sido menor. ${ }^{18}$

5. Un cambio notable, inducido también por el nuevo desarrollo, es el de la urbanización. En particular, Villahermosa y Cárdenas mostraron un proceso de crecimiento muy acelerado pues recibieron población rápidamente. Este fenómeno se debió más a las funciones centrales de comercio y servicios que requirieron las nuevas actividades económicas y la población; aunque hubo también un incremento en la actividad agroindustrial que caracteriza a Villahermosa.

Este rápido proceso de urbanización requiere de una ampliación y mejora en los servicios públicos locales, que pudieron desarrollarse mediante esfuerzos de coordinación entre las autoridades locales, estatales y federales para el desarrollo urbano del estado.

6. Por último, quiero mencionar que la actividad de PEMEX en Tabasco y en general en el sureste, ha sido criticada por el deterioro que ha causado en la ecología del trópico. Este aspecto es motivo de controversia, pues se arguye que los derrames accidentales de petróleo y las fugas de gas no utilizable afectan mínimamente el equilibrio

17 Consejo Nacional de Población, Diagnóstico y politica demográfica del estado de Tabasco, 1981 (mimeog.).

18 Entre 1959 y 1963, del total de inversión pública federal en Tabasco, $77.6 \%$ se destinó al petróleo y sólo $22.4 \%$ a otros sectores. Entre 1971 y 1976, fueron $72 \%$ destinados al petróleo y $28 \%$ al resto de los sectores, véase Consejo Nacional de Población, op. cit. 
Cuadro 6

Tabasco: Población económicamente activa por rama de actividad y grupos de ingreso, 1979* (porcentajes)

\begin{tabular}{|c|c|c|c|c|c|c|c|}
\hline & Total & $\begin{array}{l}\text { Menos del } \\
\text { salario } \\
\text { minimo }\end{array}$ & $\begin{array}{c}1 \text { a } 1.5 \text { veces } \\
\text { el salario } \\
\text { minimo }\end{array}$ & $\begin{array}{c}1.5 \text { a } 2 \text { veces } \\
\text { el salario } \\
\text { minimo }\end{array}$ & $\begin{array}{c}2 \text { a } 3 \text { veces } \\
\text { el salario } \\
\text { minimo }\end{array}$ & $\begin{array}{l}3 \text { a } 5 \text { veces } \\
\text { el salario } \\
\text { minimo }\end{array}$ & $\begin{array}{c}5 \text { a } 7 \text { veces irias } \\
\text { el salario } \\
\text { minimo }\end{array}$ \\
\hline Agricultura & 100.00 & 64.12 & 18.17 & 6.19 & 5.70 & 2.82 & 3.00 \\
\hline Transformación & 100.00 & 22.17 & 24.40 & 20.55 & 26.36 & 3.36 & 3.16 \\
\hline Construcción & 100.00 & 31.54 & 23.28 & 27.16 & 12.62 & 3.64 & 1.76 \\
\hline $\begin{array}{l}\text { Construcción } \\
\text { PEMEX }\end{array}$ & 100.00 & 10.85 & 9.95 & 15.74 & 19.83 & 14.81 & 28.82 \\
\hline $\begin{array}{l}\text { Industria } \\
\text { petrolera }\end{array}$ & 100.00 & 3.04 & 23.63 & 4.61 & 23.18 & 35.63 & 9.91 \\
\hline Servicios & 100.00 & 36.5 & 16.23 & 13.16 & 19.63 & 10.32 & 4.16 \\
\hline
\end{tabular}

Fuente: Encuesta sociodemográfica del estado de Tabasco, CONAPO-COPRODET, 1980

* El salario mínimo se consideró de 3000.00 pesos mensuales equivalentes a 43 dls. 
ecológico, sobre todo si se comparan con otras medidas como la tala masiva de la selva, la contaminación por fertilizantes, etc. ${ }^{19}$

A juzgar por el conjunto de resultados de una década de actividad petrolera, estamos ante un desarrollo que, hasta el momento, no ha sido capaz de aprovechar las nuevas actividades económicas para impulsar el crecimiento de la región. Estos rangos son similares a los encontrados en zonas de nuevas implantaciones industriales del país como Coatzacoalcos, Minatitlán, Las Truchas o Salina Cruz y se califican por quienes los han analizado, como desarrollos con características de "enclave" y no como polos de desarrollo. ${ }^{20}$

La discusión sobre si las actividades económicas, relacionadas con la explotación petrolera constituyen un polo de desarrollo para la región o tienen características de enclave, se relaciona con la aplicación de políticas de desarrollo regional. Conviene aclarar brevemente uno y otro conceptos para analizar la conveniencia de su utilización en el caso de Tabasco.

El concepto de polo de desarrollo nace originalmente con los trabajos de Perroux, ${ }^{21}$ como una explicación general a la forma en que opera el desarrollo económico y surge a partir de la observación empírica de que el desarrollo no ocurre en forma homogénea ni simultánea sobre el territorio. La aplicación del concepto de polo de desarrollo en lugares geográficos específicos (regiones) se debe a Boudeville, quien parte de la idea de una región polarizada como el área homogénea, continua, localizada en el espacio geográfico y cuyas partes son interdependientes y se relacionan alrededor de un centro regional o "polo". Este se define como un grupo de industrias en expansión, localizado en un área urbana, $\mathrm{e}$ induce a un des-

19 Véase Alejandro Toledo (coordinador). Petróleo y ecodesarrollo en el sureste de México, México, Centro de Ecodesarrollo, 1982.

20 Véase Zavala de Cossío, Ma. Eugenia. Industria peirolera y cambio sociodemográfico: Algunos efectos sobre la población del crecimiento del complejo industrial Coatzacoalcos-Minatitlán-Cozaleacoque y C. Ruiz Chiapetto, Efectos de las implantaciones industriales en el crecimiento demogrófico: lecturas criticas, ambos trabajos fueron presentados en $\mathrm{E} 1$ Coloquio Franco-Mexicano, "Los grandes complejos industriales y su impacto en el espacio latinoamericano", México, D.F., septiembre, 1981 y S. Frauvergue. Changements dands l'isthme de Tehuantepec: la raffinerie de Salina Cruz, París, Institut des Huates etudes de L'Amerique Latine, Document de Recherche núm. 3, septiembre, 1980.

21 Véase Perroux, F., L'economie du XX $X^{e}$ siecle (segunda edición), París, Presses Universitaires de France, 1964. 
arrollo ulterior de actividades económicas, a través de su zona de influencias. ${ }^{22}$

La confusión entre la teoría general del-desarrollo económico mediante polos (Perroux), y los conceptos relacionados con agrupaciones territoriales (lugares centrales, complejos industriales) su evolución y efectos (teorías de la transmisión del desarrollo económico, de la difusión geográfica de las innovaciones, de la base económica, etc.), es una de las causas de la debilidad analítica en que ha caído esta teoría. ${ }^{23}$

Las características esenciales de una implantación tipo enclave se derivan de su relación con el ámbito exterior a la región donde se localiza la actividad económica, generalmente basada en la explotación de un recurso natural, y predominantemente exportadora. Esta relación se da de tal forma, que todo efecto-inductor de desarrollo para la región sale de este ámbito.

Así, la relación de un enclave con su "metrópoli" es de subordinación total y no hay posibilidades de negociación con ésta. El resultado es que, tanto las etapas de elaboración final de los productos como los efectos multiplicadores de esta actividad se localizan fuera del enclave. A pesar de experimentar períodos de aparente auge, la región-enclave no se desarrolla y al cesar, por cualquier motivo, la explotación del recurso, experimenta una fuerte depresión. ${ }^{24}$

El caso del desarrollo generado por la explotación petrolera en Tabasco y la región ístmica, presenta características que se pueden atribuir a ambas formas de desarrollo; esto dificulta su clasificación como polo de desarrollo o enclave. La actividad productiva, si bien se concentra en la explotación-exportación del petróleo, no se ha reducido a esto pues a partir de ella se impulsa fuertemente la construcción y se pone en marcha un complejo industrial petroquímico y otras industrias afines. Este tipo de desarrollo industrial se asimila al concepto de industria "propulsiva" en un polo de desarrollo y, de hecho, ha atraido población y urbanización en las zonas donde se localiza. No obstante estas circunstancias y a juzgar por sus efectos en el empleo, distribución del ingreso, bajo nivel de inversiones locales

22 Boudeville J., Problems of regional planning, citado por H. W. Richardson, Regional Economics, New York, Praeger, 1969.

23 Véase Hermansen $T$., "Development poles and development centres in national and regional development: Elements of a theoretical fromework" en A. Kuklinski (comp.), Growth poles and growth centres in regional planning, París, Mouton, 1970.

24 C. Ruiz Chiapetto, op. cit. 
y deterioro ecológico, la situación parece más bien de enclave (véanse las páginas 95-100).

\section{¿Recuperación económica nacional o desarrollo regional en Tabasco?}

No es la intención de esta reflexión final, dar recetas sobre lo que se podría o debería hacer para evitar estas consecuencias y lograr un efecto multiplicador de significación para la economía regional. Sólo discutiré brevemente la relación entre políticas de crecimiento regional y nacional, con el fin de resaltar algunos elementos que puedan servir para entender mejor la capacidad y limitaciones reales de desarrollo en la región de Tabasco.

La descripción anterior sugiere que estamos ante una situación equiparable, a nivel nacional y a nivel de la región productora de petróleo. Si con los hidrocarburos el país enfrenta los peligros de la monoexportación, la región padece, a una escala menor, los sintomas de una actividad con características de enclave. Ambas situaciones desembocan en una dependencia con respecto al "exterior" y en una imposibilidad de internalizar los beneficios, derivados de la exportación de sus recursos naturales. La situación se agrava si tomamos en cuenta el horizonte temporal de explotación de un recurso no renovable, cuya demanda se ha elevado momentáneamente, que irá decayendo conforme se desarrollen nuevas tecnologías para la utilización de otras fuentes de energía. Esto hace más urgente el aprovechamiento de recursos en la actualidad para prever situaciones futuras a más largo plazo. El argumento se completa con la reiterada observación con respecto a la necesidad de una decisión política más firme, que oriente la evolución de la estructura productiva nacional y fomente el desarrollo a escala regional.

No obstante la validez de estos argumentos, su generalización puede ocultar aspectos importantes. El país y la región petroleras constituyen dos ámbitos socioeconómicos y políticos distintos aunque forzosamente relacionados, por lo que su carácter de productoresexportadores de petróleo no puede ofrecer las mismas posibilidades para su manejo ni tiene las mismas consecuencias. Asimismo, hay que considerar la relación que existe entre las metas de crecimiento y recuperación económica del país y las de desarrollo de una región en particular.

Con el diseño de su política energética, además de responder a 
las necesidades internas, el país debe hacer frente a un mercado internacional con características oligopólicas. En la relación externa, no estamos ante una situación al estilo colonial, donde la capacidad de negociación con la "metrópoli" era prácticamente nula. La política de ventas al ex terior es muy delicada; los errores afectan drásticamente los ingresos del país, pero al mismo tiempo se conserva cierto margen de libertad en los niveles de producción, precios, clientela, etc. El petróleo constituye, además, un arma importante en las negociaciones internacionales, en especial con Estados Unidos que es el principal comprador.

A nivel regional, el petróleo no es en absoluto un elemento de presión de la entidad frente al gobiemo central. Este recurso se maneja monopólicamente por el estado, desde su expropiación en 1938, y las decisiones en cuanto a volúmenes de extracción y explotación que afectan directamente a las regiones productoras no están en sus manos, sino en función de las metas de eficiencia nacional.

Sin embargo, en cuanto al manejo de los ingresos estatales, Tabasco tiene márgenes amplios de independencia política y económica. Cuenta también con instrumentos jurídico-administrativos para dirigir esos recursos hacia inversiones que tiendan a desarrollar las potencialidades productivas en otras ramas de actividad (véase la introducción de este artículo) y a mejorar las condiciones de empleo, educación, vivienda, servicios públicos, etcétera.

Al pensar en el impacto de la actividad petrolera sobre las economías nacional y regional, hay que tomar en cuenta las estructuras productiva y social, anteriores a la llegada de estas actividades. Esto forma parte del paisaje sobre el que debieron implantarse las nuevas actividades y seguramente influye en las posibilidades de desarrollo futuro.

Así, por ejemplo, el auge petrolero en México llega después de 40 años de industrialización, lo cual es una condición muy favorable para el desarrollo ulterior del sector. A nivel regional se encuentran, por el contrario, condiciones que favorecen la "filtración" hacia otras regiones de efectos multiplicadores de las inversiones en la zona, como una escasa diversificación industrial cuando comenzó la actividad de PEMEX. Es menor la experiencia en cuanto a calificación de mano de obra, antecedentes de inversión, actitudes empresariales, etcétera, $y$ esto implica mayores dificultades para efectuar una transición regional rảpida hacia la modernización. A pesar de que la inversión pública se incremente rápidamente, como en el caso de Tabasco, la respuesta del sector privado en cuanto a la moviliza- 
ción de sus recursos, se efectúa a un ritmo distinto. Las inversiones de capital privado en las nuevas actividades requieren también de otras condiciones y no son una respuesta automática a la inversión pública.

Si bien las metas nacionales y regionales son distintas y sus condiciones socioeconómicas eran diferentes al comienzo de la actividad relacionada con el petróleo, para evaluar sus efectos en ambos niveles hace falta considerar el tipo de relación entre los objetivos de desarrollos nacional y regional.

Un análisis de este tipo requiere de información más precisa sobre los planes e instrumentos para su aplicación en ambos niveles y rebasa el objetivo de este trabajo, sólo haré una observación general.

Los planes de desarrollo en Tabasco se fortalecieron a partir de la puesta en marcha de la explotación-exportación del petróleo, como instrumento de la recuperación económica del país, y no surgieron como requerimientos local y autónomo. El desarrollo regional de Tabasco, a pesar de los esfuerzos posteriores llevados a cabo desde el interior de la zona, fue originalmente una decisión del poder central. Esto se explica al tomar en cuenta la importancia del efecto de derrame para el país derivado de la expansión de las inversiones en esta región.

Un punto de conflicto pudo ser que las grandes inversiones en Tabasco hizo que disminuyeran en otros sitios, ${ }^{25}$ pero esto no ocurrió en las regiones generadoras de recursos (en especial divisas) para el país. Desde esta perspectiva y en la actual coyuntura, los recursos asignados a la actividad petrolera de Tabasco tuvieron efectos multiplicadores mayores para el país y la región que inversiones alternativas en otros sectores de actividad o zonas geográficas. De ahí que haya compatibilidad de intereses nacionales y regionales con respecto a las inversiones en la zona, aunque los efectos para una y otra fueron distintos y menos satisfactorios para la propia región.

25 Véase Horst Siebert, Regional Economic Growth: Theory and Policy, Scranton, Pennsylvania, International Textbook Company, 1969 (parte IV). 